\title{
Efecto Analgésico Postexodoncia Simple del Extracto de Morinda citrifolia (Noni): Ensayo Clínico Aleatorizado de Grupos en Paralelo
}

\author{
Post Exodontia Analgesic Effect of Morinda citrifolia (Noni): \\ A Randomized Clinical Trial in Parallel Groups
}

\author{
Milagros del Pilar Cornejo Ferradas; ; Angel Steven Asmat Abanto** \& Segundo Guillermo Ruíz Reyes ${ }^{\star \star *}$
}

CORNEJO, F. M. P.; ASMAT, A. A. S. \& RUíZ, R. S. G. Efecto analgésico posexodoncia simple del extracto de Morinda citrifolia (noni): ensayo clínico aleatorizado de grupos en paralelo. Int. J. Odontostomat., 8(3):433-438, 2014.

RESUMEN: El objetivo de este trabajo fue comparar el efecto analgésico post exodoncia simple del extracto de Morinda citrifolia (Noni) de $30 \mathrm{~g}$ vs. $15 \mathrm{~g}$. Este ensayo clínico paralelo aleatorizado, se desarrolló en la Clínica Estomatológica de la Universidad Privada Antenor Orrego (Trujillo, Perú). Los pacientes, quienes requerían exodoncia simple, fueron distribuidos aleatoriamente en tres grupos de 17 sujetos cada uno: Noni a dosis de $30 \mathrm{~g}$, de $15 \mathrm{~g}$ y grupo testigo (ibuprofeno). El procedimiento fue estandarizado, evaluándose el efecto analgésico mediante la Escala Visual Analógica, a las 2, 8, 24 y 48 horas posteriores a la primera toma del fármaco. El análisis estadístico se realizó mediante la prueba T de Student para comparación de medias para una $p<0,005$. No existe diferencia en el efecto analgésico post exodoncia simple entre el extracto de Noni de $30 \mathrm{~g}$ y $15 \mathrm{~g}$, a las $2(p=0,09), 8(p=0,22), 24(p=0,61)$ y 48 horas $(p=0,67)$. Ambas dosis fueron similares o superiores al control. El extracto de Noni presenta efecto analgésico post exodoncia simple.

PALABRAS CLAVE: Analgésicos, extracción dental, Morinda.

\section{INTRODUCCIÓN}

De los tratamientos quirúrgicos que realiza un odontólogo, la extracción dentaria es el procedimiento más común (Becker, 2010), y el dolor es el síntoma post exodoncia más prevalente referido por los pacientes (Pozzi \& Gallelli, 2011; Jung et al., 2005).

Morinda citrifolia (Noni), presenta bondades conocidas desde hace más de 2000 años por los habitantes de las Islas Polinesias (McClatchey, 2002; Wang et al., 2002; Pande et al., 2005; Heinicke, 1985). Noni es, sin duda, el medicamento más importante de esta región (McClatchey) y, según los estudios toxicológicos reportados, no presenta reacciones adversas (West et al., 2006; Rosly et al., 2011).

Noni se ha usado para el tratamiento de artritis, malestares estomacales, convulsiones, cuadros infecciosos, como cicatrizante y analgésico (McClatchey; Pande et al.; Basar et al., 2010). Siendo sus hojas y frutos los más empleados en la medicina tradicional (Jayaraman et al., 2008).

Las propiedades analgésicas demostradas por Noni en otros campos de la medicina, son una tentativa a la potencial introducción de la terapéutica natural al campo de la odontología. De ser eficaz, Noni podría ser empleado como analgésico alternativo, preferentemente en pacientes sistémicamente comprometidos $\mathrm{y} /$ o con alergia a los analgésicos comerciales comunes. Por este motivo, el propósito del presente trabajo fue comparar el efecto analgésico post exodoncia simple de Morinda citrifolia de $30 \mathrm{~g}$ con el de $15 \mathrm{~g}$.

\section{MATERIAL Y MÉTODO}

El presente ensayo clínico, de diseño paralelo aleatorizado, se realizó en la Clínica Estomatológica de la Universidad Privada Antenor Orrego (Trujillo Perú), entre noviembre y diciembre de 2011. Para la

Estudiante del Programa de Maestría en Estomatología. Universidad Privada Antenor Orrego, Trujillo, Perú.

* Maestro en Estomatología. Docente del Programa de Maestría en Estomatología y de la Escuela de Estomatología. Universidad Privada Antenor Orrego, Trujillo, Perú.

*** Doctor en Farmacia y Bioquímica. Docente de la Facultad de Farmacia y Bioquímica. Universidad Nacional de Trujillo, Trujillo, Perú. 
ejecución, se contó con la aprobación de la Facultad de Medicina Humana de la Universidad Privada Antenor Orrego, que considera los principios éticos de la Declaración de Helsinki y de la Ley General de Salud del Perú (Ley N²6842).

El trabajo se realizó con los pacientes atendidos durante la práctica clínica de las asignaturas de Cirugía Bucal I, Cirugía Bucal II y Clínica Integral I. La población muestral fue de 51 pacientes (17 por grupo), determinada mediante la fórmula para comparación de medias, previa prueba piloto.

Se eligieron para el estudio los pacientes ASA I de 18 a 50 años de edad, con indicación de exodoncia simple para 1 o 2 dientes adyacentes y con grado de instrucción primaria completa. Se excluyeron a los pacientes que no aceptaron participar, los alérgicos a los fármacos a usar, gestantes o mujeres en período de lactancia y aquellas que no utilizaban una forma adecuada de anticoncepción. Además se excluyeron a los pacientes que hubiesen recibido anestesia, sedantes, paracetamol, antiinflamatorios no esteroides (AINE), antidepresivos tricíclicos, opiáceos, corticosteroides, anticonvulsivantes, fenotiazinas, alcohol o cafeína 48 horas antes de la cirugía.

Una vez iniciado el estudio, la unidad de observación fue eliminada si se excedía de dos cárpules y media para el procedimiento, si el tiempo de intervención excedía de 30 minutos, si el paciente incumplió las indicaciones post exodoncia, si no acudió a la cita control, si se presentó alguna complicación como hemorragia, infección, alveolitis, lesión de tejidos blandos u óseos adyacentes, fracturas de mandíbula o de dientes adyacentes.

Mediante la revisión de la historia clínica y el análisis radiográfico se seleccionaron a los pacientes que cumplían con los criterios mencionados. De aceptar, firmaron el correspondiente consentimiento informado.

Mediante asignación aleatoria (sorteo), realizada por un colaborador independiente al estudio, los 51 pacientes fueron distribuidos equitativamente en los siguientes grupos:

Grupo 1= 17 tratados con extracto fluido del fruto de Noni a dosis de $15 \mathrm{~g}$.

Grupo 2= 17 tratados con extracto fluido del fruto de Noni a dosis de $30 \mathrm{~g}$.

Grupo 3= 17 tratados con ibuprofeno de $400 \mathrm{mg}$.
En los tres grupos, la medicación empezó 30 minutos antes de la cirugía, y continuó cada 8 horas por 2 días.

El colaborador llevó el registro de la historia clínica y la medicación para cada paciente. El investigador principal no tuvo conocimiento de la distribución, hasta finalizar las mediciones postoperatorias, momento en el que el colaborador entregó el registro para el procesamiento de datos.

El procedimiento quirúrgico fue estandarizado y ejecutado por los estudiantes de las asignaturas mencionadas, bajo estricta supervisión de los investigadores odontólogos.

Las indicaciones, verbales y por escrito, fueron las mismas para todos los pacientes: morder un rollo de gasa por un período de 30 minutos postexodoncia, alimentación líquida o blanda y fría las primeras 24 horas, no fumar ni tomar bebidas alcohólicas, evitar esfuerzo físico y realizar la higiene oral con cepillo de cerdas suaves.

Los pacientes fueron debidamente capacitados para llenar el instrumento de medición, que fue un cuestionario estructurado, donde el paciente registró la intensidad de dolor en una Escala Visual Analógica, de 0 a $100 \mathrm{~mm}$, a las 2, 8, 24 y 48 horas posteriores a la primera toma del fármaco. Esta escala es clínicamente recomendable para cuantificar la experiencia dolorosa en los pacientes (Canakci \& Canakci, 2007; FerreiraValente et al., 2011; Sanikop et al., 2011).

Siguiendo la recomendación de Joshi et al. (2000), se mantuvo en todo momento contacto telefónico con el paciente; con esto, se pudo monitorizar el cumplimiento de las indicaciones y posibles complicaciones. Además, se efectuó una visita domiciliaria a las 9 horas post exodoncia, para supervisión del tratamiento y del llenado del instrumento.

En caso de haber encontrado fracaso de la terapia con Noni, se hubiese indicado ibuprofeno, como fármaco de rescate. Sin embargo, no fue esto necesario.

Obtención del extracto fluido de Noni. Las frutas de Noni fueron recolectadas por un especialista en Farmacognosia y Farmacobotánica del Jardín Botánico de Plantas Medicinales "Rosa Elena de los Ríos Martínez" de la Facultad de Farmacia y Bioquímica de la Universidad Nacional de Trujillo. El extracto fluido se obtuvo mediante percolación fraccionada. Cada mililitro de extracto fluido equivale a $1 \mathrm{~g}$ de fruto de Noni. Luego de obtenido el extracto, se procedió a eliminar parte del 
solvente mediante rotaevaporador, disminuyendo el volumen en un $40 \%$.

Se obtuvo para el primer grupo $54 \mathrm{ml}$ de extracto fluido de Morinda citrifolia por paciente, indicándose 9 $\mathrm{ml}(15 \mathrm{~g})$ cada 8 horas por 2 días y, para el segundo grupo $108 \mathrm{ml}$ por paciente, indicándose $18 \mathrm{ml}(30 \mathrm{~g})$ cada 8 horas por 2 días.

Administración del extracto fluido. El extracto fluido fue almacenado en frascos de vidrio color ámbar, siendo administrado al sujeto en estudio con un dosificador, capacitándole, además para su autoadmi-nistración vía oral de la siguiente manera: cada dosis de extracto fluido fue vertida en una taza de agua caliente de $200 \mathrm{ml}$ aproximadamente, con el objetivo de vaporizar el etanol, y sólo la sustancia activa sea ingerida por el paciente. Se indicó esperar 5 minutos, para el enfriamiento y la ingesta de la mezcla.

Análisis estadístico. La comparación del efecto analgésico se realizó mediante la prueba T de Student para comparación de medias para una $p<0,005$, entre ambos esquemas de tratamiento y entre cada esquema con el grupo control.

\section{RESULTADOS}

La investigación incluyó un total de 58 pacientes, de los que fueron eliminados 7 por los criterios de eliminación establecidos. Por este motivo, los datos fueron obtenidos de 51 pacientes, 38 mujeres y 13 hombres (17 pacientes por grupo).

El efecto analgésico post exodoncia simple del extracto de Morinda citrifolia a dosis de $30 \mathrm{~g}$ y de $15 \mathrm{~g}$ no presentó diferencia a las 2 horas $(p=0,09), 8$ horas $(p=0,22), 24$ horas $(p=0,61)$ y 48 horas $(p=0,67)$ (Tabla I, Fig. 1).

El extracto de Morinda citrifolia a dosis de $30 \mathrm{~g}$ presenta mayor efecto analgésico post exodoncia simple que el grupo control a las 2 horas $(p=0,028), 24$ horas $(0,008)$ y 48 horas $(p=0,026)$ (Tabla II, Fig. 2$)$.

El extracto de Morinda citrifolia a dosis de $15 \mathrm{~g}$ presenta mayor efecto analgésico post exodoncia simple que el grupo control a las 24 horas $(p=0,014)$ (Tabla III, Fig. 3).

Tabla I. Comparación del efecto analgésico post exodoncia simple del extracto fluido del fruto de Morinda citrifolia a dosis de $30 \mathrm{~g}$ y de $15 \mathrm{~g}$.

\begin{tabular}{lcccccc}
\hline $\begin{array}{l}\text { Tiempo } \\
\text { (horas) }\end{array}$ & $\begin{array}{c}\text { Morinda } \\
\text { citrifolia }\end{array}$ & Media & DE & Diferencia & T & $\mathbf{p}$ \\
\hline $\mathbf{2}$ & $15 \mathrm{~g}$ & 10,000 & 10,025 & 4,88 & 1,74 & 0,09 \\
& $30 \mathrm{~g}$ & 5,118 & 5,711 & --- & --- & --- \\
\hline $\mathbf{8}$ & $15 \mathrm{~g}$ & 8,235 & 9,953 & 3,59 & 1,26 & 0,22 \\
& $30 \mathrm{~g}$ & 4,647 & 6,164 & --- & --- & --- \\
\hline $\mathbf{2 4}$ & $15 \mathrm{~g}$ & 1,588 & 2,152 & 0,35 & 0,51 & 0,61 \\
& $30 \mathrm{~g}$ & 1,235 & 1,855 & --- & --- & --- \\
\hline $\mathbf{4 8}$ & $15 \mathrm{~g}$ & 0,294 & 0,985 & 0,12 & 0,43 & 0,67 \\
& $30 \mathrm{~g}$ & 0,176 & 0,529 & --- & --- & --- \\
\hline
\end{tabular}

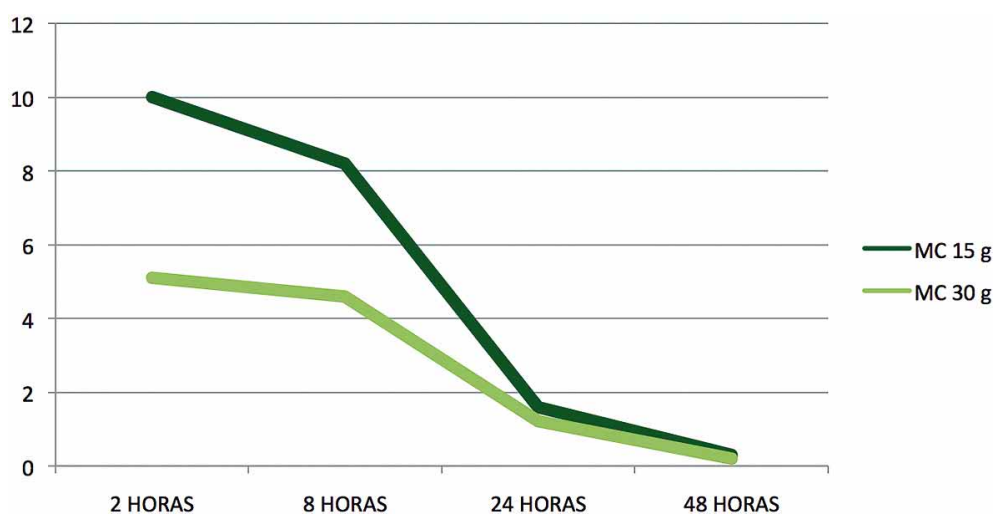

Fig. 1. Comparación del efecto analgésico post exodoncia simple del extracto fluido del fruto de Morinda citrifolia a dosis de $30 \mathrm{~g}$ y de $15 \mathrm{~g}$. 
CORNEJO, F. M. P.; ASMAT, A. A. S. \& RUíZ, R. S. G. Efecto analgésico posexodoncia simple del extracto de Morinda citrifolia (noni): ensayo clínico aleatorizado de grupos en paralelo. Int. J. Odontostomat., 8(3):433-438, 2014.

Tabla II. Efecto analgésico post exodoncia simple del extracto fluido del fruto de Morinda citrifolia a dosis de $30 \mathrm{~g}$.

\begin{tabular}{lcccccccc}
\hline & \multicolumn{4}{c}{ Morinda citrifolia $\mathbf{3 0} \mathbf{g}$} & \multicolumn{4}{c}{ Ibuprofeno } \\
\hline Tiempo & $2 \mathrm{~h}$ & $8 \mathrm{~h}$ & $24 \mathrm{~h}$ & $48 \mathrm{~h}$ & $2 \mathrm{~h}$ & $8 \mathrm{~h}$ & $24 \mathrm{~h}$ & $48 \mathrm{~h}$ \\
Media & 5,1 & 4,6 & 1,2 & 0,2 & 11,5 & 9,6 & 5,2 & 1,5 \\
DE & 5,7 & 6,2 & 1,9 & 0,5 & 9,8 & 10,9 & 5,2 & 2,1 \\
Prueba T & 2,33 & 1,63 & 2,98 & 2,44 & -- & --- & -- & -- \\
p & 0,028 & 0,117 & 0,008 & 0,026 & --- & --- & -- & -- \\
\hline
\end{tabular}

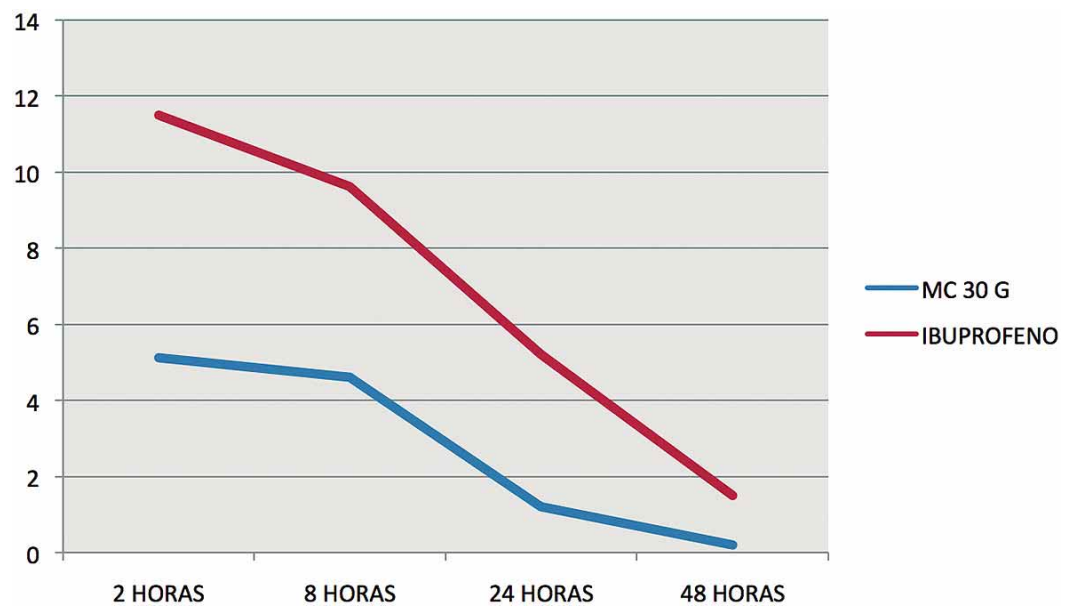

Fig. 2. Efecto analgésico post exodoncia simple del extracto fluido del fruto de Morinda citrifolia a dosis de $30 \mathrm{~g}$.

Tabla III. Efecto analgésico post exodoncia simple del extracto fluido del fruto de Morinda citrifolia a dosis de $15 \mathrm{~g}$.

\begin{tabular}{lcccccccc}
\hline & \multicolumn{4}{c}{ Morinda citrifolia 15 $\mathbf{~ g}$} & \multicolumn{4}{c}{ Ibuprofeno } \\
\hline Tiempo & $2 \mathrm{~h}$ & $8 \mathrm{~h}$ & $24 \mathrm{~h}$ & $48 \mathrm{~h}$ & $2 \mathrm{~h}$ & $8 \mathrm{~h}$ & $24 \mathrm{~h}$ & $48 \mathrm{~h}$ \\
Media & 10 & 8,2 & 1,6 & 0,3 & 11,5 & 9,6 & 5,2 & 1,5 \\
DE & 10 & 10 & 2,2 & 1,0 & 9,8 & 10,9 & 5,2 & 2,1 \\
Prueba T & 0,45 & 0,38 & 2,67 & 2,07 & --- & --- & --- & --- \\
p & 0,656 & 0,708 & 0,014 & 0,05 & --- & --- & --- & --- \\
\hline
\end{tabular}

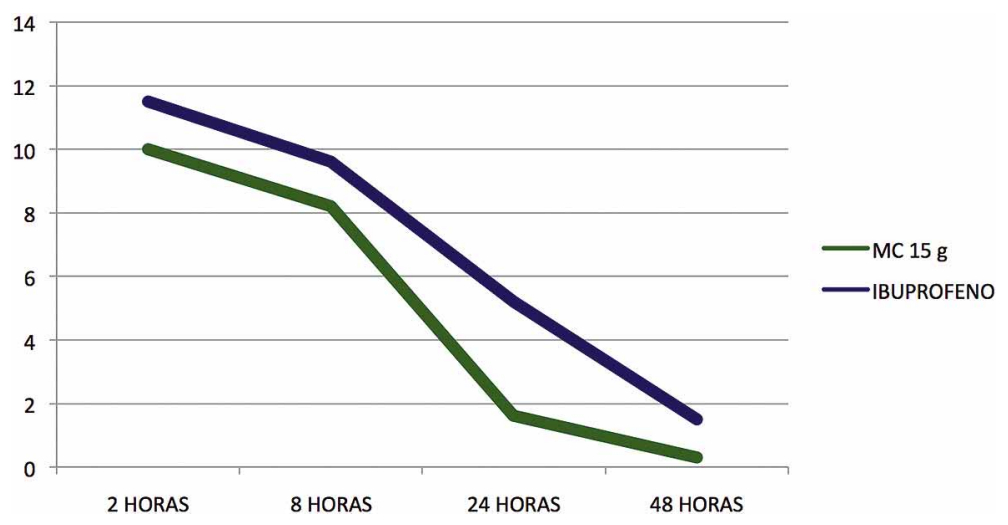

Fig. 3. Efecto analgésico post exodoncia simple del extracto fluido del fruto de Morinda citrifolia a dosis de $15 \mathrm{~g}$. 


\section{DISCUSIÓN}

En estomatología existe una gran variedad de tratamientos quirúrgicos, con diferentes modelos de dolor. Por este motivo es necesario tener diferentes alternativas para tratarlo, dando prioridad a las de menor costo y menos efectos adversos.

Entre los tratamientos quirúrgicos odontológicos, la exodoncia simple es el que más se practica en nuestro medio. Teniendo en consideración que, posterior a todo procedimiento quirúrgico bucal aparece un episodio doloroso, existe plena justificación para la utilización de medicamentos que controlan y reducen la duración y grado de dolor posoperatorio (Becker; Jung et al.; Romera et al., 2000). La evidencia de que el fruto de Noni presenta efecto analgésico proviene de experimentos en animales, realizados por Younos et al. (1990), Punjanon \& Nandhasri (2005), Akihisa et al. (2007), Deng et al. (2007) y Dussossoy et al. (2011). Según recientes investigaciones, la inhibición de las vías metabólicas de ciclooxigenasa y lipooxigenasa pueden ser las responsables de este efecto (Wang et al.; Akihisa et al.; Palu et al., 2004, 2012). Además, Younos et al., concluyeron que el fruto de Morinda citrifolia podría tener un efecto analgésico central, debido a que naloxona inhibió parcialmente su efecto.

No existen ensayos clínicos realizados con Noni para el tratamiento del dolor odontológico que nos permitan comparar los de la presente investigación; sin embargo, se puede citar el trabajo de Akimbo et al. (2006), quienes utilizaron esta planta medicinal y la fisioterapia en pacientes con espondilosis, encontrando buenos resultados en la reducción de dolor y recomendando esta terapia natural frente al uso de AINE.

Los resultados del presente estudio demuestran que no existe diferencia en el efecto analgésico post exodoncia simple entre las dosis estudiadas de Noni. Según este hallazgo, es posible utilizarlo con un potencial efecto analgésico.

Aún se desconoce la farmacocinética de Noni; sin embargo, los efectos secundarios asociados a su uso se reportan en menos del $5 \%$ de los casos. Estos incluyen eructos, diarrea y alergia. Noni ha sido reportado como seguro para embarazadas y lactantes, además puede ser ingerido con otros medicamentos y suplementos alimenticios (Basar et al.). Ninguno de los sujetos evaluados en el estudio reportó reacciones adversas. Solamente se puede mencionar como desven- taja el sabor amargo del preparado.

No debemos limitar la prescripción analgésica a los fármacos convencionales, ni prescribir basados en "nuestra experiencia". Es necesario continuar en la investigación de nuevas opciones farmacológicas, basados en ensayos clínicos bien diseñados, que permitan satisfacer los requerimientos establecidos brindar a los pacientes las mejores alternativas terapéuticas con menos reacciones adversas.

Podemos mencionar como limitante del estudio que las exodoncias fueron realizadas por los alumnos de estomatología, quienes presentan habilidades motrices distintas; sin embargo, este error se controló mediante la asignación al azar de los grupos, los criterios establecidos para elegir los casos y la estricta supervisión del procedimiento estandarizado por los investigadores, considerándose este factor como parte del error aleatorio que está presente en todas las investigaciones.

Por los resultados, se puede recomendar el uso de extracto de Noni para controlar el dolor leve-moderado post exodoncia simple. Se deben realizar más ensayos clínicos al respecto, con diferentes esquemas terapéuticos, con muestras mayores, así como trabajar en la presentación y sabor de esta sustancia, para mejorar su aceptación.

\section{AGRADECIMIENTOS}

A los docentes, estudiantes y pacientes de la Clínica Estomatológica de la Universidad Privada Antenor Orrego.

CORNEJO, F. M. P.; ASMAT, A. A. S. \& RUÍZ, R. S. G. Post exodontia analgesic effect of Morinda citrifolia (noni): a randomized clinical trial in parallel groups. Int. J. Odontostomat., 8(3):433-438, 2014.

ABSTRACT: The objective of this study was to compare the post exodontia analgesic effect of the Morinda citrifolia (Noni) extract of $30 \mathrm{~g}$ vs. $15 \mathrm{~g}$. This randomized parallel clinical trial, was developed in the Dental Clinic of Universidad Privada Antenor Orrego (Trujillo, Peru). Patients who required simple dental extraction, were randomized into three groups of 17 subjects each one: Noni at doses of $30 \mathrm{~g}, 15 \mathrm{~g}$ and control group (ibuprofen). The procedure was standardized and the analgesic effect was evaluated by Visual Analogue Scale at 2, 8,24 and 48 hours after the first dose of the drug. Statistical analysis was performed using Student 's t test for comparison of means with $p<0.005$. There is no difference in the post 
exodontia analgesic effect between Noni extract $30 \mathrm{~g}$ and $15 \mathrm{~g}$, at $2(p=0.09), 8(p=0.22), 24(p=0.61)$ and 48 hours $(p=0.67)$. Both doses were similar to or higher than control. The extract of Noni has post exodontia analgesic effect.

KEY WORDS: analgesics, tooth extraction, morinda.

\section{REFERENCIAS BIBLIOGRÁFICAS}

Akihisa, T.; Matsumoto, K.; Tokuda, H.; Yasukawa, K.; Seino, K.; Nakamoto, K.; Kuninaga, H.; Suzuki, T. \& Kimura, Y. Antiinflammatory and potential cancer chemopreventive constituents of the fruits of Morinda citrifolia (Noni). J. Nat. Prod., 70(5):754-7, 2007.

Akimbo, S. R. A.; Noronha, C. C.; Okanlawon, A. O. \& Denesi, M. A. Comparative study of the effect of Morinda citrifolia (Noni) with selected physiotherapy modalities in the management of patients with cervical spondylosis. Niger. J. Health Biomed. Sci., 5(2):6-11, 2006.

Basar, S.; Uhlenhut, K.; Högger, P.; Schöne, F. \& Westendorf, J. Analgesic and antiinflammatory activity of Morinda citrifolia L. (Noni) fruit. Phytother. Res., 24(1):38-42, 2010.

Becker, D. E. Pain management: Part 1: Managing acute and postoperative dental pain. Anesth. Prog., 57(2):67-78, 2010.

Canakci, V. \& Canakci, C. F. Pain levels in patients during periodontal probing and mechanical non-surgical therapy. Clin. Oral Investig., 11(4):377-83, 2007.

Deng, S.; Palu, K.; West, B. J.; Su, C. X.; Zhou, B. N. \& Jensen, J. C. Lipoxygenase inhibitory constituents of the fruits of noni (Morinda citrifolia) collected in Tahiti. J. Nat. Prod., 70(5):85962, 2007.

Dussossoy, E.; Brat, P.; Bony, E.; Boudard, F.; Poucheter, P.; Mertz, C.; Giaimis, J. \& Michel, A. Characterization, anti-oxidative and anti-inflammatory effects of Costa Rican noni juice (Morinda citrifolia L.) J. Ethnopharmacol., 133(1):108-15, 2011.

Ferreira-Valente, M. A.; Pais-Ribeiro, J. L. \& Jensen, M. P. Validity of four pain intensity rating scales. Pain, 152(10):2399-404, 2011.

Heinicke, R. The pharmacologically active ingredient of Noni. Pac. Trop. Bot. Gard. Bull., 15:10-4, 1985.

Jayaraman, S. K.; Manoharan, M. S. \& Illanchezian, S. Antibacterial, Antifungal and Tumor cell suppression potential of Morinda citrifolia fruit extracts. Int. J. Integr. Biol., 3(1):44-9, 2008.

Joshi, A.; Snowdon, A. T.; Rood, J. P. \& Worthington, H. V. Pain control after routine dento-alveolar day surgery: a patient satisfaction survey. Br. Dent. J., 189(8):439-42, 2000.

Jung, Y. S.; Kim, M. K.; Um, Y. J.; Park, H. S.; Lee, E. W. \& Kang, J. $W$. The effects on postoperative oral surgery pain by varying NSAID administration times: comparison on effect of preemptive analgesia. Oral Surg. Oral Med. Oral Pathol. Oral Radiol. Endod., 100(5):559-63, 2005.
McClatchey, W. From Polynesian healers to health food stores: changing perspectives of Morinda citrifolia (Rubiaceae). Integr. Cancer Ther., 1(2):110-20, 2002.

Palu, A. K.; Su, C.; Zhou, B. N. \& Jensen, J. Morinda citrifolia L: a dual inhibitor of COX-2 and 5-LOX En-zymes. San Francisco, 5th International Conference and Exhibition on Nutraceuticals and Functional Foods, 2004.

Palu, A. K.; West, B. J. \& Jensen, C. J. Noni seed oil topical safety, efficacy, and potential mechanisms of action. J. Cosmet. Dermatol. Sci. Appl., 2(2):74-8, 2012.

Pande, M.; Naiker, M.; Mills, G.; Singh, N. \& Voro, T. The Kura files: qualitative social survey. Pac. Health Dialog., 12(2):85-93, 2005.

Pozzi, A. \& Gallelli, L. Pain management for dentists: the role of ibuprofen. Ann. Stomatol. (Roma), 2(3-4 Suppl.):3-24, 2011.

Punjanon, T. \& Nandhasri, P. Analgesic effect of the alcoholic extract from the fruits of Morinda citrifolia. Acta Hortic., 678(4):103-6, 2005.

Romera, E.; Perena, M. J.; Perena, M. F. \& Rodrigo, M. D. Neurofisiología del dolor. Rev. Soc. Esp. Dolor, 7 (Suppl. 2):117, 2000.

Rosly, S. M.; Shanmugavelu, S.; Murugaiyah, M.; Hadijah, H.; Tarmizi, A. S.; Noridayusni, Y. \& Subramaniam, K. Subchronic oral toxicity study of Morinda citrifolia (Mengkudu) in spraque Dawley rat. J. Trop. Agric. Food Sci., 34(2):341-9, 2011.

Sanikop, S.; Agrawal, P. \& Patil, S. Relationship between dental anxiety and pain perception during scaling. J. Oral Sci., 53(3):341-8, 2011.

Wang, M. Y.; West, B. J.; Jensen, C. J.; Nowicki, D.; Su, C.; Palu, A. K. \& Anderson, G. Morinda citrifolia (Noni): a literature review and recent advances in Noni research. Acta Pharmacol. Sin., 23(12):1127-41, 2002.

West, B. J.; Jensen, C. J.; Westendorf, J. \& White, L. D. A safety review of Noni fruit juice. J. Food Sci., 71(8):R100-6, 2006.

Younos, C.; Rolland, A.; Fleurentin, J.; Lanhers, M. C.; Misslin, R. \& Mortier, F. Analgesic and behavioural effects of Morinda citrifolia. Planta Med., 56(5):430-4, 1990.

Dirección para Correspondencia:

Angel Steven Asmat Abanto

Maestro en Estomatología

Docente del Programa de Maestría en Estomatología y de la Escuela de Estomatología

Universidad Privada Antenor Orrego

Trujillo - PERÚ

Email: aasmata1@upao.edu.pe

Recibido : 04-03-2014

Aceptado: 25-07-2014 